\title{
Computer simulation of periodic nanostructures
}

\author{
E. N. Grishanov ${ }^{1}$, I. Y. Popov ${ }^{2}$ \\ ${ }^{1}$ National Research Ogarev Mordovia State University, \\ 68 Bolshevistskaya Str., Saransk 430005, Republic of Mordovia, Russia \\ ${ }^{2}$ ITMO University, Kronverkskiy, 49, St. Petersburg, 197101, Russia \\ grishanoven@math.mrsu.ru, popov1955@gmail.com
}

\section{DOI 10.17586/2220-8054-2016-7-5-865-868}

\begin{abstract}
An algorithm and code for spectrum calculation for periodic nanostructures in homogeneous magnetic field are developed. The approach is based on the zero-range potentials model. The mathematical background of the model is based on the theory of self-adjoint extensions of symmetric operators.
\end{abstract}

Keywords: Periodic nanostructure, MPI, parallel computing.

Received: 11 September 2016

Revised: 18 September 2016

In memory of B. S. Pavlov (1936-2016)

\section{Introduction}

Rapid development of research in the field of nanostructures and complex molecules is not possible without computer modeling and simulations. Over the last two decades, a few algorithms and codes have appeared for computer modeling of such systems. These approaches allow researchers to predict properties of nanosystems and to construct nanostructures and molecules with predetermined properties without expensive experimentation. Some of the most popular programs of such type are HyperChem [1], Gamess [2], Gasussian and Molden.

In most cases, codes for nanostructure calculations do not use the direct solution to the Schrödinger equation due to difficulties (or even impossibility) of obtaining explicit formulas and numerical approach complications. Even the use of the Hartree-Fock-Roothaan method in many cases cannot give results in a timely fashion. The complexity of calculations growths exponentially if the number of atoms in the basic cell increases. One faces this problem when calculating the spectrum of periodic arrays of quantum dots or nanotubes, graphene, etc.

This paper is devoted to describing the algorithm and code for the spectrum calculations of periodic nanostructures in an homogeneous magnetic field [3], based on the operator extensions theory [4,5]. This approach leads to an explicitly solvable model.

\section{Model construction}

Let us construct a model of spinless charged particle of mass $m$ and charge $e$ in a $2 D$-periodic nanostructure with the Bravais lattice $\Lambda$ in a homogeneous magnetic field $\mathbf{B}$. Let $K$ be a set of atoms in the basic cell of $\Lambda$. The whole structure is described by the set:

$$
\Gamma=\Lambda+K=\{\kappa+\lambda: \kappa \in \mathrm{K}, \lambda \in \boldsymbol{\Lambda}\} .
$$

Consider the free particle magnetic Hamiltonian (Landau operator),

$$
H_{0}=\frac{\hbar}{2 m}\left(\mathbf{p}-\frac{e}{c} \mathbf{A}(\mathbf{r})\right)^{2}
$$

where $\mathbf{p}=i \hbar \nabla$ is the momentum operator in $\mathbf{R}^{3}, \mathbf{A}(\mathbf{r})=\frac{1}{2} \mathbf{B} \times \mathbf{r}$ is the vector potential of the field $\mathbf{B}$ in symmetric gauge. The Hamiltonian of the particle in $2 D$-periodic lattice is constructed as a perturbation of operator (1) by zero-range potentials posed at nodes of lattice $\Gamma$. This can formally be written as:

$$
H=H_{0}+\sum_{\lambda \in \mathbf{\Lambda}} \sum_{\mathbf{k} \in \mathbf{K}} \hat{\alpha}_{k} \delta(\mathbf{r}-\lambda-\mathbf{k})
$$

where $\hat{\alpha}_{k}$ related to the interaction of the particle with the atom posed at $\mathbf{k} \in K$. If the structure is homogeneous, then $\hat{\alpha}_{\mathbf{k}}=\hat{\alpha}$ for any $\mathbf{k} \in K$. Examples of such structures include graphene and carbon nanotubes. 
We use "restriction-extension" procedure (see, e.g., [6-8]). Consider symmetric operator $S$, being a restriction of $H$ on the space of functions from $D\left(H_{0}\right)$ are vanishing at $\gamma \in \Gamma$. We seek the model Hamiltonian as a self-adjoint extension of the operator $S$. Resolvents of such operators are described by Krein's formula:

$$
R_{A}(\zeta)=R^{0}(\zeta)-\Gamma(\zeta)[Q(\zeta)-A]^{-1} \Gamma^{*}(\bar{\zeta})
$$

where $\Gamma(\zeta)$ is Krein $\Gamma$-function, $Q(\zeta)$ is the $Q$-function, correspondingly. Self-adjoint operator $A$, defined in the space of boundary values (the dimension of the space coincides with the deficiency index of $S$ ), parameterize selfadjoint extension $H_{A}$ of $S$. We choose a diagonal $A$ Relation [9] leads to an expression of the Green function $G_{A}$ of $H_{A}$ :

$$
G_{A}\left(\mathbf{r}, \mathbf{r}^{\prime} ; \zeta\right)=G_{0}\left(\mathbf{r}, \mathbf{r}^{\prime} ; \zeta\right)-\sum_{\gamma, \gamma^{\prime} \in \boldsymbol{\Gamma}}[Q(\zeta)-A]_{\gamma, \gamma^{\prime}}^{-1} G_{0}(\mathbf{r}, \gamma ; \zeta) G_{0}\left(\gamma^{\prime}, \mathbf{r}^{\prime} ; \zeta\right)
$$

Here:

$$
\begin{gathered}
G_{0}\left(\mathbf{r}, \mathbf{r}^{\prime} ; \zeta\right)=\Phi\left(\mathbf{r}, \mathbf{r}^{\prime}\right) F_{1}\left(\mathbf{r}-\mathbf{r}^{\prime} ; \zeta\right)=\Phi\left(\mathbf{r}, \mathbf{r}^{\prime}\right) F_{2}\left(\mathbf{r}-\mathbf{r}^{\prime} ; \zeta\right) \\
\Phi\left(\mathbf{r}, \mathbf{r}^{\prime}\right)=\frac{m}{2 \hbar^{2}} \sqrt{\frac{\xi}{\pi}} \exp \left[-\pi i \xi\left(\mathbf{r} \times \mathbf{r}^{\prime}\right)-\pi \xi\left(\mathbf{r}_{\perp}-\mathbf{r}_{\perp}^{\prime}\right)^{2} / \mathbf{2}\right] \\
F_{1}(\mathbf{r} ; \zeta)=\sum_{\ell=0}^{\infty} \frac{\exp \left[-\sqrt{4 \pi \xi(\ell+1 / 2)-\zeta}\left|\mathbf{r}_{\|}\right|\right]}{\sqrt{\ell+1 / 2-\zeta / 4 \pi \xi}} L_{\ell}\left(\pi \xi \mathbf{r}_{\perp}^{2}\right), \\
F_{2}(\mathbf{r} ; \zeta)=\frac{1}{\sqrt{\pi}} \int_{0}^{\infty} \frac{\exp \left[-\pi \xi\left(\mathbf{r}_{\perp}^{2} /\left(e^{t}-1\right)+\mathbf{r}_{\|}^{2} / t\right)\right]}{\left(1-e^{-t}\right) \exp [(1 / 2-\zeta / 4 \pi \xi) t]} \frac{d t}{\sqrt{t}}
\end{gathered}
$$

In (5-7), $\mathbf{r}_{\perp}$ is a projection of $\mathbf{r}$ on the plane of lattice $\Lambda, \mathbf{r}_{\|}=\mathbf{r}-\mathbf{r}_{\perp}, L_{\ell}(x)$ is the Laguerre polynomial, $\xi$ is the density of the magnetic field $\mathbf{B}$ flux in units of flux quanta $\Phi_{0}=2 \pi \hbar c /|e|$. The $Q$-function in (4) has the form of matrix $Q(\zeta)=\left(Q\left(\gamma, \gamma^{\prime}\right)\right)_{\gamma, \gamma^{\prime} \in \boldsymbol{\Gamma}}$ with entries:

$$
Q\left(\gamma, \gamma^{\prime} ; \zeta\right)=\left\{\begin{array}{l}
G_{0}\left(\gamma, \gamma^{\prime} ; \zeta\right), \quad \gamma \neq \gamma^{\prime} \\
\frac{m}{2 \hbar^{2}} \sqrt{\frac{\xi}{\pi}} Z\left(\frac{1}{2}, \frac{1}{2}-\frac{\zeta}{4 \pi \xi}\right), \quad \gamma=\gamma^{\prime}
\end{array}\right.
$$

where $Z(s, v)$ Hurvitz $\zeta[10]$.

The spectrum of $H_{A}$ consists of two parts: the spectrum of $H_{0}$ (well-known) and points in which the operator $Q(\zeta)-A$ is not invertible or its inverse operator is bounded.

It is known ( [11]) that for rational number of the magnetic flux $\eta=N / M$ values that $(N \in \mathbf{Z}, M \in \mathbf{N})$ does not change $\Lambda$ when seeking of the spectrum for $H_{A}$. The spectrum is determined by:

$$
\operatorname{det}[\widetilde{Q}(\mathbf{p} ; \zeta)-\widetilde{A}(\mathbf{p})]=0,
$$

for each $\mathbf{p} \in \mathbf{T}_{\eta}^{2}=[0,1 / M) \times[0,1)$. Matrices $\widetilde{Q}(\mathbf{p} ; \zeta)$ is known and:

$$
\begin{gathered}
\widetilde{Q}\left(\mathbf{p} ; m, \kappa ; m^{\prime}, \kappa^{\prime} ; \zeta\right)=\exp \left[\pi i\left(m-m^{\prime}\right) \xi\left(\kappa \times \mathbf{a}_{2}\right)\right] \times \\
\sum_{\lambda_{1}, \lambda_{2}=-\infty}^{\infty} Q\left(\lambda_{1} \mathbf{a}_{1}+\left(\lambda_{2} M+m-m^{\prime}\right) \mathbf{a}_{2}+\kappa, \kappa^{\prime} ; \zeta\right) \times \\
\exp \left\{\pi i \xi\left[\kappa \times\left(\lambda_{1} \mathbf{a}_{1}+\lambda_{2} M \mathbf{a}_{2}\right)\right]-2 \pi i\left(\lambda \mathbf{p}+\frac{\eta}{2} \lambda_{1}\left(\lambda_{2} M+m+m^{\prime}\right)\right)\right\},
\end{gathered}
$$

$\kappa, \kappa^{\prime} \in \mathrm{K}$ и $m, m^{\prime}=0, \ldots M-1$.Matrix $\widetilde{A}(\mathbf{p})$ is block-diagonal with identical $|K| \times|K|$ blocks. The diagonal block is in turn diagonal with constants characterizing the point-like interactions as the diagonal entries.

For each $\mathbf{p} \in \mathbf{T}_{\eta}^{2}$, equation (9) has $|K| M$ solutions. The continuous branches of equation (9) solutions $z_{l}(\mathbf{p})$ $(l=1, \ldots,|K| M)$ give one the bands of the operator spectrum. The dispersion equation can be solved numerically. 


\section{Code description}

The most interesting problem in the field is construction of "flux-energy" diagrams, i.e. to find the spectrum for each magnetic flux value $\eta$. The most remarkable diagram of such type is "Hofstadter butterfly" [12]. To construct the diagram, it is necessary to solve equation (9) for different $\eta$ values. For each $\eta$, we should find $|K| M$ bands. Calculations for different $\eta$ and different bands are independent, and can be performed simultaneously, i.e., it is natural to use parallel computation methods. Independence of computing with respect to the data allows one to use interface MPI [13]. Testing showed that organization of the following parallelization using of OpenMP is not useful and leads to decreased calculation performance due to increase processor cores cache-misses.

The initial data for the code are:

- basic vectors of the Bravais lattice $\Lambda$,

- coordinates of atoms from $K$, belonging to basic cell of the lattice $\Lambda$,

- "interaction constants" for each atom from $K$,

- vector $\mathbf{B}$ of the applied magnetic field.

The following instruments were used: compiler Free Pascal, libraries AlgLib and MPI Chamelion. Such a choice allows one to use clusters of various architecture, controlled by operational systems such as Microsoft Windows HPC Server, Linux, FreeBSD.

After initiation, the code, using MPI, creates one control and a few calculating processes. The control process reads (from an input file) values of $\eta=N / M$ and the bands numbers, distributes those among calculating processes and then collects the results. The calculating processes receive tasks from the control and send results to the control. For data exchange, the blocking functions MPI_Send and MPI_Recv are used. Due to the small amount of data transfer, the speed of calculations is, really, independent on the interconnection and grows linearly with respect to the number of cores used.

The code was used for for computing the spectrum of multi-layered graphene in a magnetic field [14] and periodic arrays of nanotubes. The results are in agreement with other models [15]. In the case of nanotube array calculations, the code works with $|K|=224$ carbon atoms in a basic cell. Using of codes analogous to HyperChem, leads to operation with hundreds of basic cells (correspondingly,tens of thousands atoms), and it gives one only small part of the periodic array. Our approach gives an essential acceleration but, of course, cannot simulate edges of the real nanostructure.

\section{Acknowledgements}

This work was partially financially supported by the Government of the Russian Federation (grant 074U01), by Ministry of Science and Education of the Russian Federation (GOSZADANIE 2014/190, Projects No 14.Z50.31.0031 and No. 1.754.2014/K), by DFG Grant NE 1439/3-1, by grant 16-11-10330 of Russian Science Foundation. The authors thanks National Research Ogarev Mordovia State University for the possibility to carry out calculations.

\section{References}

[1] Howard A., McIver J., Collins J. HyperChem. Computational Chemistry. Hypercube, 1996, 350 p.

[2] Butyrskaya E.V. Computational Chemistry: Theoretical Foundation and Using of Codes Gaussian and Gauss View. M.: Solon Press, 2011, $224 \mathrm{p}$.

[3] Grishanov E.N. Code for spectrum calculation for periodic nanostructures in a magnetic field. Certificate of state registration of code No 2016618953 from 10.08.2016.

[4] Geyler V.A., Pavlov B.S., Popov I.Yu. One-particle spectral problem for superlattice with a constant magnetic field. Atti. Sem. Mat. Fis. Univ. Modena., 1998, 46, P. 79-124.

[5] Grishanov E.N., Eremin D.A., Ivanov D.A., Popov I.Yu., Smirnov P.I. Periodic chain of disks in a magnetic field: bulk states and edge states. Nanosystems: Phys. Chem. Math., 2015, 6(5), P. 637-643.

[6] Albeverio S., Kurasov P. Singular perturbations of differential operators. Solvable Schrödinger type operators. London Mathematical Society Lecture Notes 271. Cambridge Univ. Press. Cambridge, 2000.

[7] Pavlov B.S. The theory of extensions and explicity-solvable models. Russ. Math. Surv., 1997, 42(6), P. $127-168$.

[8] Albeverio S., Fassari S., Rinaldi F. Spectral properties of a symmetric three-dimensional quantum dot with a pair of identical attractive d-impurities symmetrically situated around the origin. Nanosystems: Phys. Chem. Math., 2016, 7(2), P. 268-299.

[9] Geyler V.A., Demidov V.V. Spectrum of three-dimensional Landau operator perturbed by a periodic point potential, $T M F$, 1995, 103(2), P. 283--294.

[10] Bateman H., Erdélyi A. Higher transcendental functions, V.I. McGraw-Hill, New York, 1953. 
[11] Geyler V.A. The two-dimensional Scrödinger operator with a uniform magnetic field, and its perturbation by periodic zero-range potentials. St. Petersburg Math. J., 1992, 3, P. 489-532.

[12] Hofstadter D.R. Energy levels and wave functions of Bloch electrons in rational and irrational magnetic fields. Phys.Rev. B., 1976, 14, P. 2239-2249.

[13] Pacheco Peter S. Parallel Programming With MPI, Morgan Kaufmann, 1997, 418 p.

[14] Grishanov E.N., Popov. I.Yu. Spectral properties of multi-layered graphene in a magnetic field. Superlattices and Microstructures, 2015, 86, P. 68-72.

[15] Nemec N., Cuniberti G. Hofstadter butterflies of bilayer graphene. Phys. Rev. B (Rapid Comm.), 2007, 75, P. 201404(R). 